\title{
Barley alloplasmic lines - the spectra of peculiar plasmon types
}

Siniauskaya M.*, Lukhanina N., Makarevich A., Pankratov V., Liaudansky A., Goloenko I., Shymkevich A., Danilenko N., Davydenko O.

Institute of Genetics and Cytology, NASB, Minsk, Belarus

*e-mail: cytoplasmic@mail.ru

The coordination of nuclear and organelle genomes is substantial for functioning of green plants, especially for coadaptation of plants to different environmental conditions. Intensive use of next generation sequencing technologies for nuclear genomes exploration allowed to accumulate the tremendous amount of data about various organisms. Organellar genomes are studied not so intensively. Alloplasmic lines are the suitable model for evaluation of functioning the same nucleus on different cytoplasms, revealing the effects of nuclear-cytoplasmic interactions. The collection of alloplasmic lines of barley Hordeum vulgare with cytoplasm of Hordeum spontaneum was created and studied in our laboratory in the pre-NGS time. At present we performed the isolation of chloroplast and mitochondrial DNA of 12 samples from this collection, and sequenced them on Illumina MiSeq. Quite novel data were obtained concerning the variability of Hordeum chloroplast and mitochondrial genomes. Totally 6 indels, 58 SNP, 15 SSR in cpDNA were detected between studied set of samples. The most important data were verified by Sanger sequencing. Concerning the plastid DNA, the alloplasmic lines with W8 cytoplasm and different nucleus grouped in one subcluster. The lines with W3 and W4 cytoplasms demonstrated dissimilarities in clusterization depending on the definite nucleus genomes, varying cpDNA types were detected. Mitochondrial genome sequencing revealed similar trend, but the level of variability was much lower. The cause of observed differences in the organelle genomes of alloplasmic lines with the cytoplasm from the same source and different nucleus is not clear. Probably it is the initial heterogeneity of cytoplasms in the samples of wild barley taken for creation of alloplasmic lines or it alternatively may be the outcome of some organelle genome modifications during the coexistence of a new combination of nucleus and cytoplasm. So, next generation sequencing of organellar DNAs gives new breakthrough for analysis of the chloroplast and mitochondrial genomes. Revealing the organellar variability facilitates further development of our knowledge about nuclear-cytoplasmic interactions. 